\title{
Is Irritable Bowel Syndrome over Diagnosed in Duhok
}

\section{City Community-Iraq?}

\author{
Sefar Mohamad Haj ${ }^{1}$ and Waleed Jameel Omar Barwari ${ }^{2}$ \\ 1. Department of Medicine, Faculty of Medical Sciences, School of Medicine, Duhok University, Iraq \\ 2. Department of Microbiology, Faculty of Medical Sciences, School of Medicine, Duhok University, Iraq
}

\begin{abstract}
The symptoms of IBS are common in community, there are overwhelming numbers of Diagnosed cases of IBS in Duhok Governorate, and frequently many cases appears to have been misdiagnosed. This study is through light on the very update methods of diagnosing IBS, its pathophysiology with the most update treatment. All diagnosed cases of IBS for more than three months, both in azadi General Hospital Out Patient Department and Private clinics are re-evaluated for the possibility of misdiagnosis and all were fully investigated for all differential diagnosis and according to its clinical presentation by most recent methods available for each disease. The aim of this study is to: (1) Know the prevalence of IBS in Dohuk Governorate and to be compared with studies done in other localities in different countries. (2) Differentiate between patients who are really IBS from those due to other causes and wrongly diagnosed as IBS. (3) Stimulate other doctors to investigate all suspected cases and not to depend merely on clinical presentation. The results shows that most cases are diagnosed according to clinical presentation which is important but not enough at all and the diseases should be diagnosed after excluding all diseases with similar presentation and not to miss any treatable conditions. The total number of the IBS patients were 529 (76.01\%), while the number of the patients that misdiagnosed as IBS were 167 (23.99\%) due to other causes that needed investigations. The differences among IBS, sex, and other causes were significant at $(\mathrm{p}<0.095)$. The percentage of cigarette smokers $52.9 \%$ was significantly associated with IBS The study shows high percentage of misdiagnosed treatable cases.
\end{abstract}

Key words: IBS, over diagnosed, bowel, community.

\section{Introduction}

It is a functional colonic disorder characterized by chronic abdominal pain or discomfort, bloating, flatulence and alteration of bowel habit, constipation or diarrhoea may predominate, or they may alternate in the absence of any detectable organic cause. In some cases the symptoms are relieved by bowel movements $[1,2]$. Irritable bowel syndrome (IBS) has been considered a diagnosis of exclusion; however, it is no longer considered a diagnosis of exclusion, but it does have a broad differential diagnosis [3]. Irritable bowel syndrome is one of the most common gastro-intestinal disorders in developed countries with prevalence estimated between $10 \%$ and $20 \%$. Currently the diagnosis of irritable bowel syndrome is based on the

Corresponding author: Sefar Mohamad Haj, MD, lecturer, research fields: headache and toxoplasmosis. E-mail: sm_haj@yahoo.com.
Rome III diagnostic criteria. These criteria state that in order for a diagnosis of IBS to be given, patients must satisfy the following:

At least 3 months, with onset at least 6 months previously of recurrent abdominal pain or discomfort associated with 2 or more of the following:

(1) It is relieved with defecation, and/ or

(2) Onset is associated with a change in frequency of stool, and/ or

(3) Onset associated with a change in form (appearance) of stool.

Due to general nature of these symptoms (which can occur in numerous with other conditions) IBS is often only diagnosed after lengthy process of eliminating during which the patient may undergo a number of unnecessary and often invasive test to rule out other diseases [4, 5]. Kruis score [6] Manning criteria [7], IBS Jennifer criteria (www.ibsjennifer.com) also could be used to diagnose IBS. The Red flag or alarm signs 
should be excluded, and include no abnormal physical finding or history pathognomonic of other diseases, normal ESR\& WBC, no anemia or history of blood in the stool (IBS Jennifer Red flag criteria). Pathogenesis is likely multifactorial in nature. The proposed biopsychosocial model for IBS which views IBS as a product of the cumulative interactions between physiological, psychosocial, behavioural, and environmental factors seems especially appropriate in this context [8]. Recent findings appear to suggest that disruption of serotonergic equilibrium may have a role in IBS which include increased postprandial levels of circulating 5-HT in patients with diarrhea-predominant IBS, elevated platelet-depleted plasma 5-HT levels in both fasting and fed states in patients with diarrhoea-predominant-IBS, lack of elevation in plasma 5-HT after meal ingestion in patients with constipation-predominant IBS, and decreased mucosal 5-hydroxyindoleacetic acid (5-HIAA)/5-HT ratio in those with Constipation predominant-IBS [9-11]. The possible role of genetics and putative susceptibility loci for IBS has been an area of growing investigation and interest. Multiple family studies have consistently reported that familial aggregation occurs in IBS [12, 13]. The antibiotic rifaximin was found to relieve symptoms of IBS for weeks after stopping the drug. Patients given the medication reported less bloating, abdominal pain and relief from loose. These findings confirm that bacteria flora in the gut play a key role in the development of irritable bowel syndrome [14, 15]. Normally, colony counts of gram-positive bacteria and fungi in the duodenum and jejunum are less than $1 \times 10^{5}$ organisms/mL [16, 17].

\section{Methods}

From May 2007 to July 2011, 696 patients ( 360 male and 336 female) were diagnosed to have IBS for more than 3 months were re-evaluated, the patients included those attending the out patients department of Azadi Teaching Hospital or attending the private clinic. All were on treatment for IBS including anticholinergics, antidiarrhoeals, tricyclic antidepressant, serotonin (5-HT3) Receptor antagonist, antibiotics, and bulk forming laxatives for more than 3 months. The ages were between 16-74 years. All were on normal diet with various occupations. A detailed history was taken including cigarettes smoking, alcohol intake and drugs specially those causing diarrhea (Digoxin, laxative, Colchicine...etc) and those causing constipation (calcium antagonist, tricyclic antidepressant, narcotics, iron...etc). A detailed medical history was taken for all patients like bloody diarrhoea, evidence for steatorrhoea, and history of milk intolerance.

All patients were re-examined for pulse rate, flushing, tremors, hyper pigmentations and signs of systemic diseases. All patients had full clinical examination to see if there are any signs of systemic diseases and investigations were directed according to the findings. In IBS, routine clinical tests yield no abnormalities. General stool examination 3 times 2 days apart was done for all patients to detect the trophozoites or cysts of Giardia lamblia or Entameba histolytica, and to detect the parasitic ova. Stool for occult blood, Complete Blood Count \& ESR was done for all. Colonoscopy was done for all patients with Red Flag signs that include: abnormal mass on abdominal examination, anemia, high ESR, high WBC count, Positive Occult blood test and loss of weight. 24 hours stool fat estimation for those with steatorrhoea. Antibodies screen for celiac disease (anti-endomysial antibodies) and then endoscopy with 6 duodenal or jejunal biopsies for all positive cases [18]. Serum cortisol was done for those with hyper pigmentation. TSH was done for those with constipation. FT3, FT4 and TSH were done for those patients with diarrhea and signs that suggest thyrotoxicosis. Tropical sprue was suspected following exclusion of all causes of malabsorption. All positive cases were treated according to the diagnosis and cured cases are considered misdiagnosed. Jejunal aspirate for bacterial count was done if Bacterial overgrowth syndrome was suspected [16, 17]. 


\section{Results}

The investigations of 167 (79 male and 88 female) patients were abnormal (Figs. 1and 2). There were 50 patients with chronic giardiasis and the general stool examination reveal giardial cysts and they respond to Metronidazole, those not responding to Metronidazole did respond to Albendazole or Tinidazole. There were 45 patients with chronic amoebiasis and general stool examination reveals either Entameba histolytica trophozoites or Cysts along with positive occult blood stool test. Those patients with cyst and negative stool for occult blood were common but they were not included. Those patients with Entameba histolytica trophozoite and did not respond to Metronidazole + Diloxanide furate were probably due Entameba dispar and other non pathogenic entamebae and they were excluded.

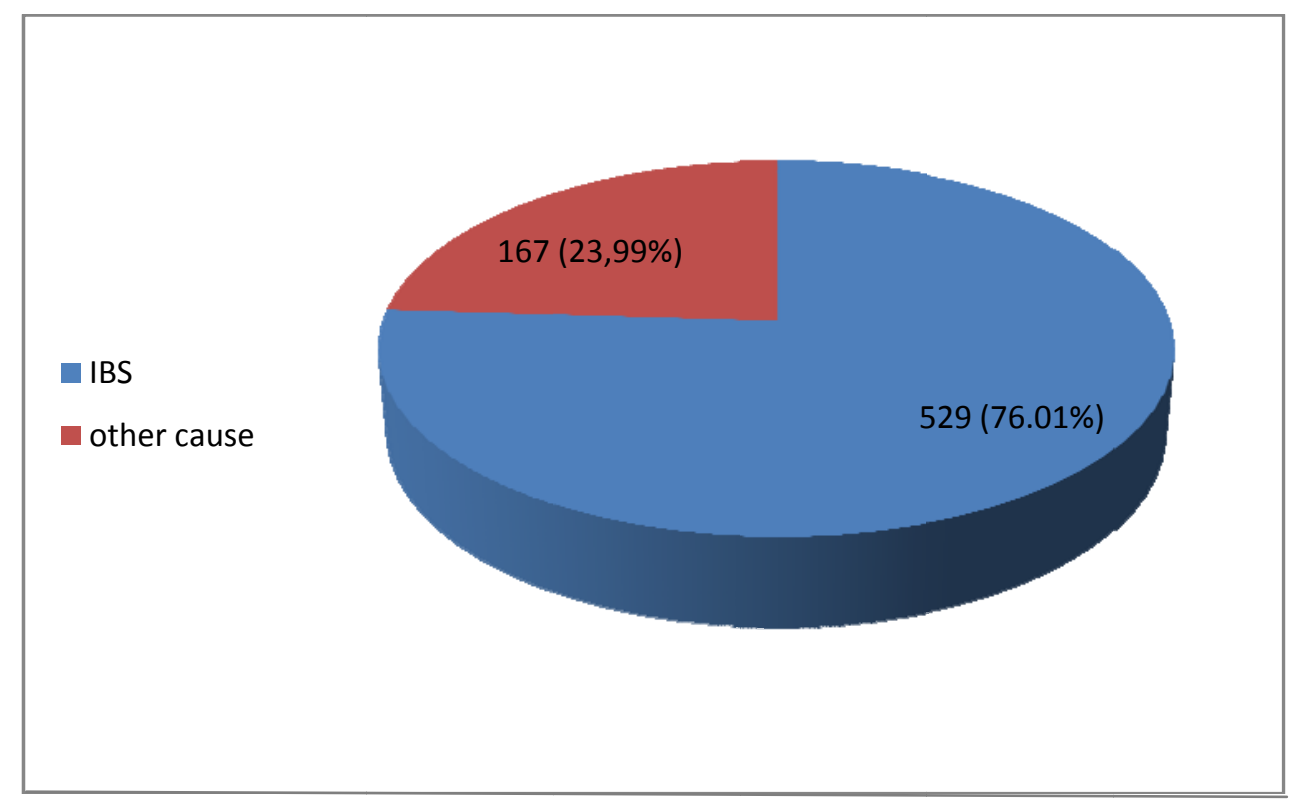

Fig. 1 The prevalence of IBS.

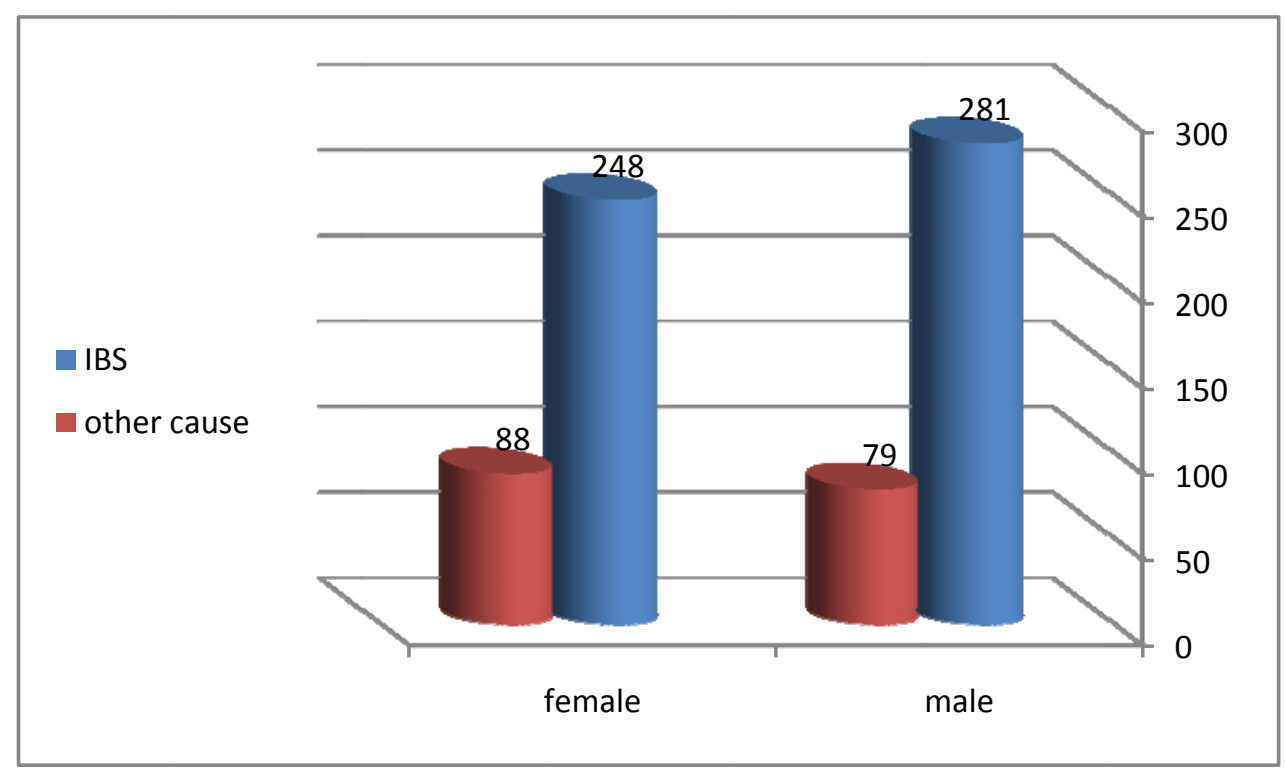

Fig. 2 Gender variation in IBS and other causes. 
Thyroid function tests were abnormal 14 patients 8 with thyrotoxicosis (predominantly diarrhea) and 6 with hypothyroidism (predominantly constipation). All respond to proper treatment. In 12 patients stool examination reveals Hymenolepis nana ova, all patients were young with ages between 16-25 years and were cured with treatment. In 8 patients duodenal or jejunal biopsies shows complete villous atrophy and they responded to gluten free diet, and they were diagnosed to have Coeliac disease. There was lactose intolerance in 14 patients and they were improved after reducing or Restriction the milk intake [19]. In 16 patients there was large number of bacteria in the stool or in the jejunal aspirate and was due to Bacterial overgrowth syndrome and they respond to proper antibiotics, nutritional support. In 7 patients, there was partial villous atrophy in duodenal biopsy and they responded to treatment of Tropical sprue, that is Tetracycline plus Folic acid, B12 \& Iron for 30 days [20]. In one patient Crohn's disease was diagnosed. Most of the IBS patients were cigarette smokers 280 (52.9\%).

\section{Discussion}

The study found that most patients were diagnosed to have IBS on clinical ground alone without satisfying any diagnostic criteria especially Rome III criteria [4, 5] and in most patients no investigations were done at all apart from general stool examination in some patients. That is way the total number of diagnosed cases were high 696 and the number of missed diagnosed patients were high too (167). This mean that $23.99 \%$ of patients were miss diagnosed, which is very high by any standard. Most of the undiagnosed cases were common and endemic diseases in this area such as giardisis in 50 cases, chronic amoebiasis in 45 cases, Hymenolepis Nana in 12 patients. There were 14 cases of Lactose intolerance indicating that proper history was not taken in detail [19]. Thyroid abnormality was common as there were 8 patients with thyrotoxicosis presenting with diarrhoea and 6 patients with hypothyroidism presenting with constipation. Lactose intolerance which is secondary to low levels of lactase enzyme in the brush border of the duodenum [21] is more common in Asian country than European country was found in 12 patients [22]. Coeliac disease was diagnosed in 8 patients which is one of the differential diagnosis of IBS according to NICE criteria and should be excluded prior to IBS diagnosis [5]. Tropical sprue which is rather common in tropical area presenting with diarrhoea was diagnosed in 7 patients [20]. Bacterial overgrowth syndrome was diagnosed in many patients \& was over diagnosed in 16 patients [16, 17]. There was one case of Crohn's diseases which is a rather uncommon disease in Iraq in comparison with Europe and North America [23].

The total number of the IBS patients were 529 (76.01\%) which is much higher than the prevalence of IBS in Shiraz city of Iran 10.9\% [24]; and in AlJouf city of Saudi Arabia 9.2\% [25], while the number of the patients that misdiagnosed as IBS were 167 (23.99\%) due to other causes that needed investigations.

The differences among IBS, sex, and other causes were significant at $(\mathrm{p}<0.095)$.

The percentage of cigarette smokers $52.9 \%$ was significantly associated with IBS and this agrees with the study done in Japan by Fujiwara [26].

\section{References}

[1] E. A. Mayer, Clinical practice. Irritable bowel syndrome, N. Engl. J. Med. 358 (16) 2008) 1692-1699.

[2] M. W. Schmulson and L. Chang, Diagnostic approach to the patient with irritable bowel syndrome, Am. J. Med. 107 (5A) (1999) 20S-26S.

[3] B. M. Spiegel, M. Farid, E. Esrailian, J. Talley and L. Chang, Is irritable bowel syndrome a diagnosis of exclusion? A survey of primary care providers, gastroenterologists, and IBS experts, Am J Gastroenterol. 105 (4) (2010) 848-858.

[4] G. F. Longstreth, W. G. Thompson and W. D. Chey et al., Functional bowel disorders, Gastroenterology 130 (2006) 1480-1491.

[5] The National Institute for health and Clinical Exellence (NICE) new guideline for the diagnosis and management of Irritable Bowel Syndrome, Mathew Hogg BSC, 4 Mar. 2008. 
[6] W. Kruis, C. Thiem and M. Weinzierl et al., A diagnostic score for the irritable bowel syndrome: Its valuate in the exclusion of organic disease, Gastroenterology 87 (1984) 1-7.

[7] A. P. Manning, W. G. Thompson and K. W. Heaton et al., Towards positive diagnosis of the irritable bowel, Br Med J 2 (1978) 653-654.

[8] [8] A. Halpert and D. Drossman, Biopsychosocial issues in irritable bowel syndrome, J Clin Gastroenterol 39 (2005) 665-669

[9] W. Atkinson, S. Lockhart and P. J. Whorwell et al., Altered 5-hydroxytryptamine signaling in patients with constipation- and diarrhea-predominant irritable bowel syndrome, Gastroenterology 130 (2006) 34-43.

[10] S. P. Dunlop, N. S. Coleman and E. Blackshaw et al., Abnormalities of 5-hydroxytryptamine metabolism in irritable bowel syndrome, Clin Gastroenterol Hepatol 3 (2005) 349-357.

[11] L. A. Houghton, W. Atkinson and R. P. Whitaker et al., Increased platelet depleted plasma 5-hydroxytryptamine concentration following meal ingestion in symptomatic female subjects with diarrhoea predominant irritable bowel syndrome, Gut 52 (2003) 663-670.

[12] G. R. Locke, A. R. Zinsmeister and N. J. Talley et al., Familial association in adults with functional gastrointestinal disorders, Mayo Clin Proc 75 (2000) 907-912.

[13] Y. A. Saito, G. M. Petersen and J. J. Larson et al., Familial aggregation of irritable bowel syndrome: A family case-control study, Am J Gastroenterol 105 (2010) 833-841.

[14] Mark Pimentel, GI Motility, N Engl J Med 364 (2011) 22-32.

[15] M. Pimentel, E. L. Chow and H. C. Lin, Normalization of lactulose breath testing correlates with symptoms improvement in irritable bowel syndrome: A double-blind, randomized, placebo-controlled study, Am J Gastroenterol 98 (2) (2003) 412-419.
[16] J. Bures, J. Cyrany and D. Kohoutova et al., Small intestinal bacterial overgrowth syndrome, World J Gastroenterol. 16 (24) (2010) 2978-2990.

[17] E. M. Quigley and A. Abu-Shanab, Small intestinal bacterial overgrowth, Infect Dis Clin North Am. 24 (4) (2010) 943-959, viii-ix.

[18] [18] A. Tursi, G. Brandimarte and G. M. Giorgetti, Prevalence of antitissue transglutaminase antibodies in different degrees of intestinal damage in celiac disease, $\mathrm{J}$ Clin Gastroenterol. 36 (3) (2003) 219-221.

[19] S. Mishkin, Dairy sensitivity, lactose malabsorption, and elimination diets in inflammatory bowel disease, Am J Clin Nutr. 65 (2) (1997) 564-567.

[20] R. C. Clark and J. Katz, Ttopical sprue emedicine Medscape, article 182986, Jan. 3, 2010.

[21] T. H. Vesa, P. Marteau and R. Korpela, Lactose intolerance, J Am Coll Nutr. 19 (2 Suppl) (2000) 165S-175S.

[22] A. D. Newcomer, D. B. McGill and P. J. Thomas et al., Tolerance to lactose among lactase-deficient American Indians, Gastroenterology 74 (1) (1978) 44-46.

[23] A. Kornbluth, D. B. Sachar and P. Salomon, Crohn's disease, in: M. Feldman, B. F. Scharschmidt and M. H. Sleisenger (Eds.), Sleisenger \& Fordtran's Gastrointestinal and Liver Disease: Pathophysiology, Diagnosis, and Management (vol 2, 6th ed.), Philadelphia, Pa: WB Saunders Co, 1998, 1708-1734.

[24] F. Khademolhosseini, D. Mehrabani and M. Nejabat et al., Irritable bowel syndrome in adult over 35 years in Shiraz, southern Iran: prevalence and associated factors, J Res Med Sci 16 (2) (2011) 200-206.

[25] A. H. Alhazmi, Irritable bowel syndrome in secondary school male students in Aljouf Province, north of Saudi Arabia, J Pak Med Assoc. 61 (11) (2011) 1111-1115.

[26] Y. Fujiwara, M. Kubo and Y. Kohata et al., Cigarette smoking and its association with overlapping gastroesophageal reflux disease, functional dyspepsia, or irritable bowel syndrome, Intern Med. 50 (21) (2011) 2443-2447. 